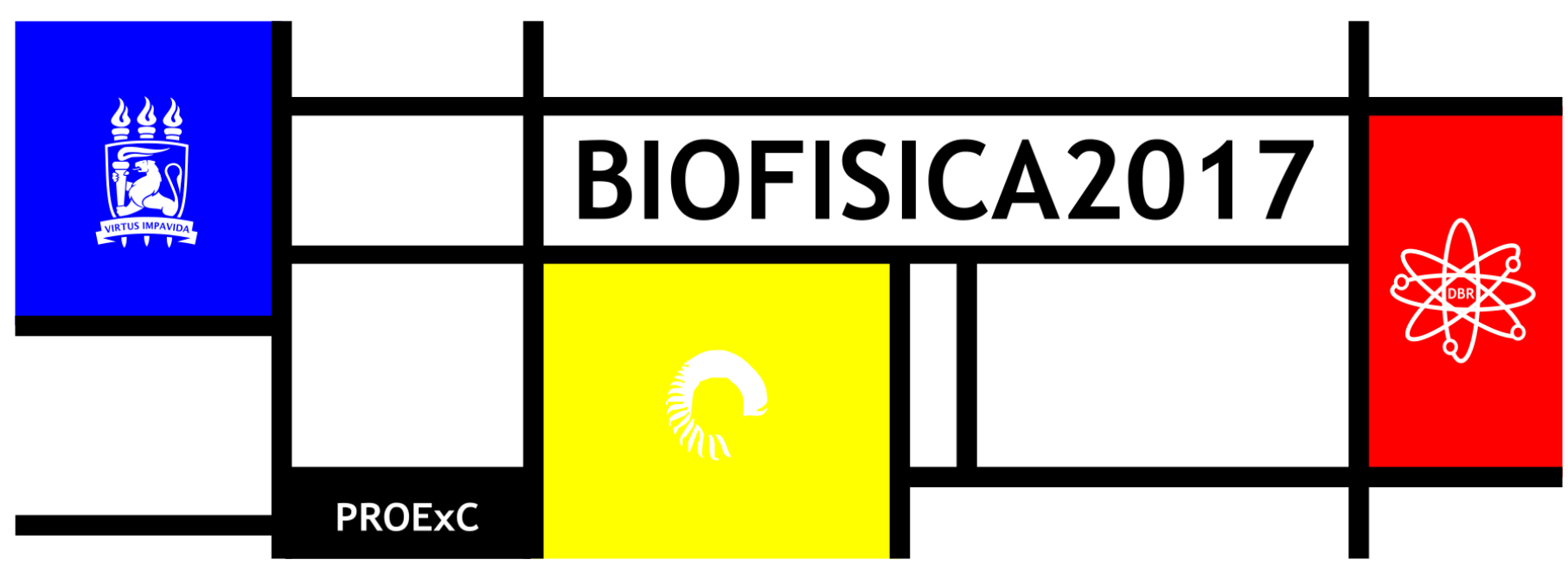

\title{
ANÁLISES MATEMÁTICAS DA ATIVIDADE ELETRICA CEREBRAL DE RATOS EXPOSTOS A RADIAÇÃO IONIZANTE
}

\author{
Camilla de Andrade Tenorio Cavalcanti ${ }^{1 *}$, Isvânia Serafim da Silva Lopes ${ }^{2}$, Leandro Álvaro de Alcantara Aguiar ${ }^{1}$, \\ Maria Teresa Jansem de Almeida Catanho ${ }^{2}$, Alexandre Parísio Barbosa de Oliveira ${ }^{3}$, Romildo Albuquerque Nogueira ${ }^{1}$ \\ ${ }^{1}$ Laboratório de Biofísica Teórico-Experimental e Computacional, UFRPE; ${ }^{2}$ Laboratório de Biofísica Celular e Molecular, UFPE; ${ }^{3}$ Instituto de \\ Radioterapia Waldemir Miranda. \\ *camillat.bio@gmail.com
}

\section{INTRODUÇÃOO}

A radioterapia craniana é uma modalidade de tratamento importante na gestão dos tumores primários e metastáticos do cérebro, leucemia e linfomas. Entretanto, essa modalidade de terapia pode ocasionar alterações cerebrais que geram danos ao indivíduo, incluindo, cognitivos (GARCIA, 2015; ANDRES-MACH, 2008; MONJE, 2003; LOGANOVSKY, 2001). Além disso, a exposição do indivíduo à radiação ionizante $(\mathrm{RI})$ pode causar efeitos diretos e indiretos nas estruturas vivas. Os efeitos indiretos resultam em radicais livres, que são bastante reativos, podendo interferir com o metabolismo de proteínas, dos lipídios e carboidratos, e quanto aos efeitos diretos, eles ocorrem quando a radiação é absorvida diretamente por moléculas importantes no metabolismo celular, como enzimas e o DNA (GARGIA, 2015).

H. Davis e P. Davis (1939) foram os primeiros pesquisadores que utilizaram o eletroencefalograma (EEG) para estudar os efeitos das radiações ionizantes sobre o cérebro. Em seus estudos, eles encontraram significativas mudanças na atividade elétrica em macacos expostos à radiação, enquanto que, Yaar e colaboradores (1982) mostraram que doses de radiação ionizante entre 1,5 e 4 Sv podem ocasionar um aumento das ondas beta no EEG em homens e mulheres.

Pesquisas demostram que as células neurogênicas localizadas na zona subgranular (SGZ), que dão origem aos neurônios do giro denteado do hipocampo (CHEN, 2008), são sensiveis à radiação ionizantes, pois doses que variam entre 0,4 Gy e 18 Gy podem levar a apoptose dessas células. Após 48 horas da exposição à radiação ionizante é possível observar uma redução da proliferação de células, da diferenciação celular e aumento da apoptose, que podem persistir durante meses (ANDRES-MACH, 2008; MONJE, 2003). Alguns estudos apoiam a hipótese de que essa diminuição da neurogênese contribui para os déficits cognitivos apresentados por pacientes após a radioterapia craniana (MONJE, 2003). Mesmo pequenas doses, podem afetar vias neurais envolvidas no envelhecimento normal, bem como nas vias envolvidas na doença de Alzheimer. Também podem ocasionar mudanças no perfil de expressão do gene, e respostas neuroinflamatórias (LOGANOVSKY, 2009).

Em trabalhos experimentais a atividade elétrica cortical pode ser obtida diretamente do córtex do animal, com uma técnica conhecida como eletrocorticograma $(E C o G)$, enquanto que no eletroencefalograma (EEG) os sinais cerebrais são capturados por meio de eletrodos posicionados no couro cabeludo, o que caracteriza uma forma não invasiva. 0 eletrocorticograma (ECoG) é uma metodologia de registro da atividade elétrica do córtex cerebral, a qual utiliza eletrodos fixados diretamente na superfície exposta do córtex. O ECoG registra a atividade na superfície ou no interior do córtex cerebral, portanto é mais invasivo do que o EEG, porém traz informações com maior precisão (SANTOS, 2007; CARDOSO, 2005).

Métodos matemáticos têm sido usados na análise dos registros do EEG e do ECoG (AGUIAR et al, 2015), dentre esses métodos a complexidade de Lempel-Ziv tem sido usada para investigar a complexidade do sinal do EEG e ECoG (PESSOA et al, 2015; AVILOV; POPOV, 2014). Portanto, esses métodos serão de grande utilidade para investigar possíveis alterações causadas pela radiação ionizante no cérebro.

Com base no exposto, esse trabalho busca analisar, por meio de métodos matemáticos, possíveis alterações na atividade elétrica em ratos expostos a RI, procurando compreender, de modo associativo, os efeitos das radiações ionizantes em humanos que passaram por uma radioterapia.

\section{MATERIAIS E MÉTODOS}

3.1. Grupos Experimentais

Foram utilizados doze ratos da linhagem Wistar (Rattus norvegicus, var. albinus), com 60 dias de idade, provenientes do biotério do Departamento de Morfologia e Fisiologia Animal da Universidade Federal Rural de Pernambuco (UFRPE), com aprovação da Comissão de Ética no Uso de Animais sob Protocolo: 23082.006238/2016-57. Os animais foram alojados em gaiolas, mantidos em ambiente adequado com temperatura $\left(23 \pm 2{ }^{\circ} \mathrm{C}\right)$ e umidade controlada, em ciclo claro-escuro de 12 horas, com acesso a água e a dieta 
especifica. Os doze ratos foram divididos em dois grupos $(n=6)$ : grupo 1 (controle) animais normais não expostos a radiação e o grupo 2 animais expostos a radiação ionizante (RI) analisados com 24h e 30 dias após a irradiação.

\subsection{Exposição à Radiação}

Os animais foram irradiados no Instituto de Radioterapia Waldemir Miranda (IRWAM), Recife-PE, Brasil. Para tal, os mesmos, foram anestesiados com xilazina $(10 \mathrm{mg} / \mathrm{kg})$ e quetamina $(75 \mathrm{mg} / \mathrm{kg})$ via intraperitoneal (IP), e em seguida foram posicionados na mesa de um acelerador linear Varian, modelo Clinac 600C (FIG. 1). A dose utilizada foi de 18 Gy de raios- $X$, de forma que os raios foram colimados para que somente a região de cabeça/pescoço fossem irradiadas, sendo metade aplicados na parte superior ( $9 \mathrm{~Gy}$ ) e a outra na parte inferior da cabeça ( $9 \mathrm{~Gy}$ ). A taxa de dose utilizada foi de $2,4 \mathrm{~Gy} / \mathrm{min}$ a $1,5 \mathrm{~cm}$ de profundidade, sendo o tempo total de exposição de 5,02 minutos.

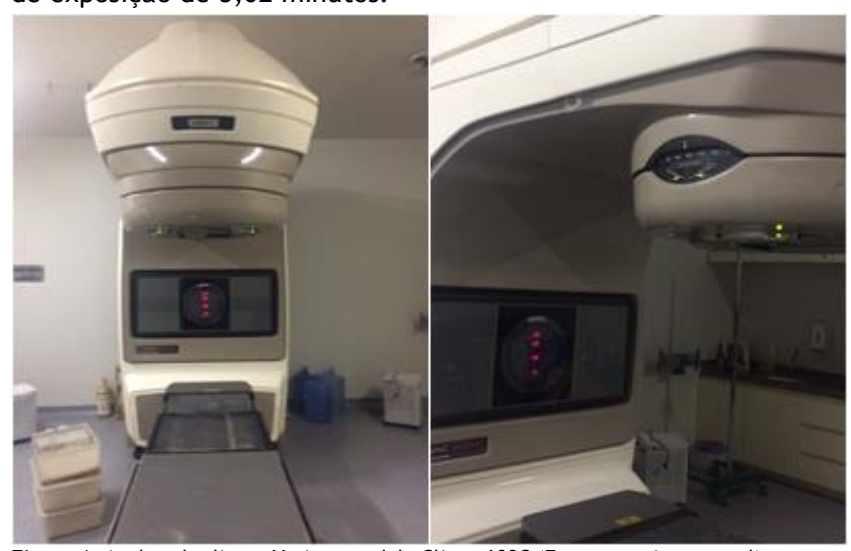

Figura 1. Acelerador linear Varian, modelo Clinac 600C (Fonte: arquivo pessoal).

\subsection{Procedimento cirúrgico para implante dos eletrodos}

Aos 60 dias de idade, os animais foram anestesiados com xilazina (10 $\mathrm{mg} / \mathrm{kg})$ e quetamina $(75 \mathrm{mg} / \mathrm{kg}$ ) via intraperitonial. Após a tricotomia e assepsia do campo cirúrgico os animais tiveram suas cabeças fixadas à base de um aparelho estereotáxico (Insight ${ }^{\circledR}$ ). Em seguida uma incisão longitudinal da pele, na linha média do crânio foi realizada e o periósteo removido. Com auxílio de um micromotor foram feitas duas perfurações, uma na região parietal e outro na região nasal. Nessas pequenas perfurações foram fixados dois microparafusos de aço inox, utilizados como eletrodos de registro. Para fixação dos parafusos foi utilizada resina acrílica e em seguida a pele foi suturada, expondo apenas os parafusos que serviram de eletrodos. Durante cinco dias, após o implante foi aplicado o antibiótico tópico Rifamicina.

\subsection{Registro da atividade elétrica cerebral}

Após sete dias da cirurgia de implante do eletrodo e verificação da completa cicatrização da cirurgia, cada animal teve a atividade elétrica cerebral registrada por 20 min com um aparelho EMG 410C (ENG System, Brasil) utilizando uma taxa de amostragem de 750 $\mathrm{Hz}$. Os registros foram antes da exposição à RI, 24h e 30 dias após a exposição.

\subsection{Análise dos registros da atividade elétrica cerebral}

Os registros foram segmentados em janelas e 2 minutos. Esses segmentos foram importados para o programa Matlab 7.8 (Mathworks, Natick, MA, USA), no qual foi implementado o algoritmo para o cálculo do espectro de potência das ondas cerebrais do ECoG, por meio da Análise de Fourier, como também foi realizada a Complexidade de Lempel-Ziv (CLZ).

\subsubsection{Análise de Fourier}

A Transformada de Fourier (TF) é uma análise direta da série temporal que permite passar a informação no domínio do tempo para o domínio da frequência, desta forma, torna possível o conhecimento da contribuição de cada componente de frequência presente numa serie temporal (WEISSTEIN, 2004).

\subsubsection{Complexidade de Lempel-Ziv (CLZ)}

A análise da CLZ baseou-se na definição descrita por Lempel-Ziv (1976), posteriormente discutida por Kaspar e Schuster (1987) para análise de padrões em séries espaço-temporais discretas. Seus valores normalmente estão entre 0 e 1 . Esta abordagem transforma o sinal analisado em uma sequência de dados binária. Este processo inicial é realizado a partir do estabelecimento de uma média do segmento analisado. Uma nova sequência ( $S$ ) é construída usando a média do segmento original como limiar. Se o valor da amostra é maior que a média, é definido como um (1), de outra maneira é zero (0). Logo, S é composta apenas por estes dois valores binários. É realizada uma varredura em $\mathrm{S}$ de sua primeira amostra até o fim. Quando é descoberta uma nova subsequência, que não foi encontrada no processo de varredura anterior, o valor da complexidade é aumentado em um (1). Assim, a CLZ simboliza o número de todas as subsequências diferentes contidas na sequência original. Um fluxograma do algoritmo pode ser visto em Kedadouche et al (2015). Em geral, a complexidade C(n) normalizada é bastante usada para se obter uma medida independente do comprimento de sequência.

$$
C L Z=C(n) / b(n) ; b(n)=N / \log 2(N) \text {; }
$$

A CLZ é uma medida de complexidade que tem sido usada para analisar os sinais de EEG em pacientes com a Doença de Alzheimer, Transtorno do Déficit de Atenção com Hiperatividade (TDAH), medir o aprofundamento de anestesia, epilepsia dentre outras condições (SIMONS; ABASOLO; HUGHES, 2015; LEE et al., 2014; IBÁÑNEZ-MOLINA et al. 2015). Ela é essencialmente uma medida de imprevisibilidade de uma série, mas que pode sofrer influência do conjunto de frequências que compõe o sinal.

\subsection{Análise estatística.}

0 teste estatístico de Kruskal-Wallis com o post-hoc de Dunn foi usado na análise dos valores obtidos para CLZ.

\section{RESULTADOS E DISCUSSÃO}

Dos pacientes tratados com radioterapia, 95\% desenvolvem alguma forma de reação na pele (SCHINEIDER, 2012) e foi observado o surgimento de radiodermite nos animais (FIG. 2) logo após uma semana da exposição à RI. A radiodermite trata-se de um conjunto de lesões cutâneas provocadas por uma exposição excessiva à RI, cuja ocorrência está limitada ao campo de tratamento com radiação (SCHINEIDER, 2012). A radiodermite é um dos efeitos adversos da radiação que são normalmente reversíveis e resolvidas espontaneamente (GREENE-SCHOLOESSER, 2012). Nos animais expostos a RI, a radiodermite desapareceu cerca de uma semana após o surgimento.

Com o espectro de potência do ECoG foi possível identificar as variações entre as ondas cerebrais dos animais do grupo controle, e aqueles analisados com 24 horas (RI24h) e o com 30 dias (RI30d) após a irradiação. Estudos já comprovaram que o padrão do eletroencefalograma (EEG) em humanos exposto a radiação ionizante são bastantes distintos se comparado com o padrão dos indivíduos normais, podendo observar o aumento da atividade das ondas cerebrais beta e delta juntamente com a diminuição de alfa e teta (LOGANOVSKY, 2001). Nos animais expostos a RI foi observado alterações nos padrões do ECoG já 24 horas após a irradiação, que foram ainda mais marcantes ao serem analisados com trinta dias (FIG 3.).

Uma análise quantitativa foi também realizada para avaliar a ação da radiação ionizante na atividade elétrica cerebral. Esta análise foi a Complexidade Lempel-Ziv (CLZ), que já vem sendo utilizado para analisar sequência de nucleotídeos em DNA e sinais neuronais (NUNES, 2014). Após calcular os valores da CLZ utilizando o MATLAB, foi construído um gráfico comparativo entre o grupo controle, RI24h e RI30d (FIG. 4). 


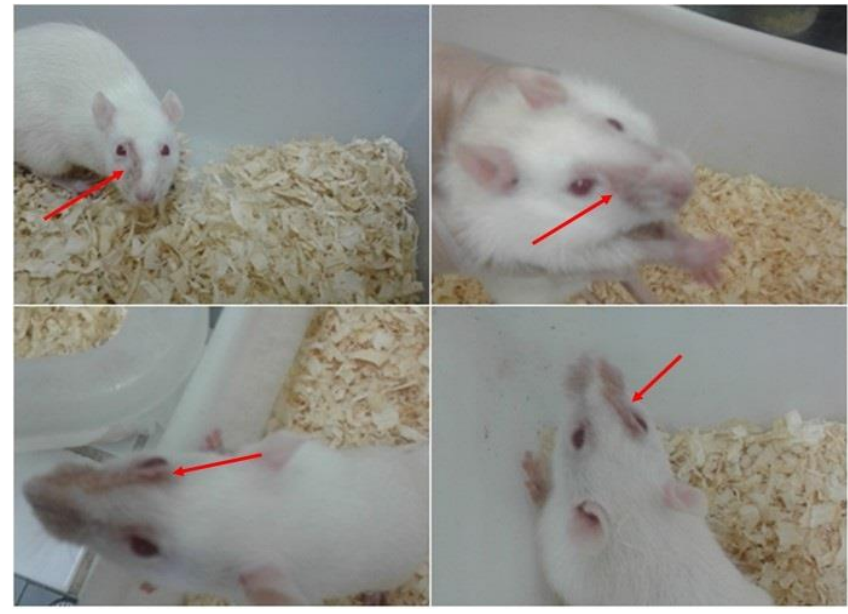

Figura 2. Radiodermite aos $\mathrm{X}$ dias após exposição à $\mathrm{RI}$, indicada pelas setas vermelhas. (Fonte: arquivo pessoal).

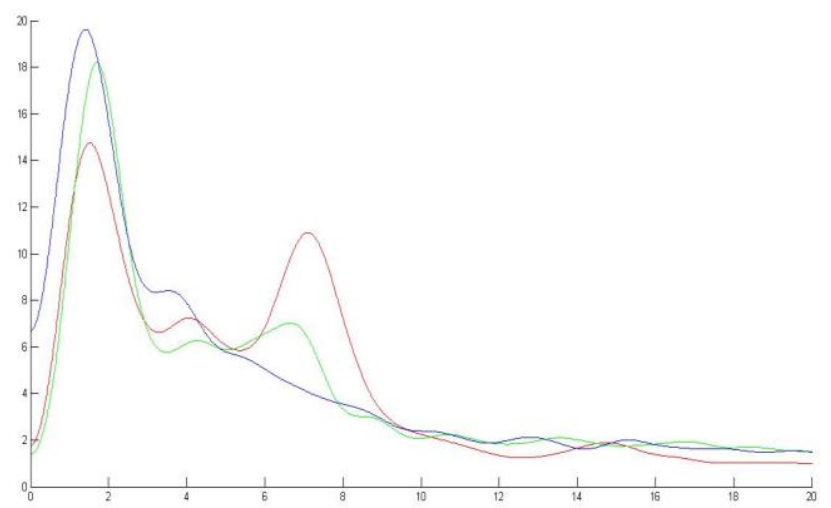

Figura 3. Espectro de Potência do ECoG do grupo controle (vermelho), RI24h (verde) e RI30d (azul). As ondas cerebrais Delta $(0,5-4 \mathrm{~Hz})$, Teta $(4-8 \mathrm{~Hz})$, Alfa $(8-12 \mathrm{~Hz})$ e Beta $(12-32 \mathrm{~Hz})$ (Fonte: arquivo pessoal).

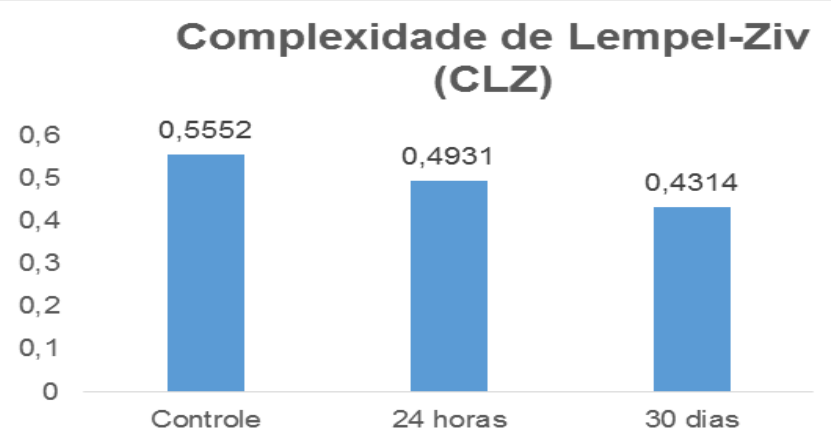

Figura 4. Análise da Complexidade de Lempel-Ziv dos animais do grupo controle, RI24h e RI30d.

A análise mostrou que os valores de CLZ diferiram do controle somente após 30 dias da exposição à radiação, ao nível de significância 0,039. O resultado da CLZ mostra que alterações observadas no sinal do ECoG é um efeito tardio da irradiação.

\section{CONCLUSÕES}

Através das análises feitas nos animais expostos a radiação ionizante pode-se concluir que os efeitos surgem logo após 24 horas, com a ocorrência de mudanças significativas nos padrões do ECoG em relação aos animais do grupo controle, tendo alterações nos padrões de todas as ondas cerebrais (delta, alfa, teta e beta). Após uma semana da irradiação foi visualizado o surgimento da radiodermite, um dos principais efeitos adversos causados pela RI, mas que foi tratado espontaneamente e desapareceu com cerca de uma semana após o surgimento. Depois de 30 dias de irradiação foram realizados novos registros do ECoG e pôde ser observado alterações no padrão da atividade elétrica cerebral. A
Complexidade de Lempel-Ziv (CLZ) mostrou que a atividade elétrica do cérebro nos animais irradiados diferiu do controle somente após 30 dias da exposição à radiação. O resultado da CLZ mostra que alterações observadas no sinal do ECoG é um efeito tardio da irradiação.

\section{REFERÊNCIAS}

AGUIAR, L. A. A; SILVA, I. M. S; FERNANDES, T. S; NOGUEIRA, R. A. Long-term correlation of the electrocorticogram as a bioindicator of brain exposure to ionizing radiation. Brazilian Journal of Medical and Biological Research, v. 48, n. 10, p 915 - 922; 2015. ANDRES-MACH, M; ROLA, R; FIKE, J. R. Radiation effects on neural precursor cells in the dentate gyrus. Cell and tissue research, $v$. 331, n. 1, p. 251 - 262, jan. 2008.

AVILOV, O.; POPOV, A. Different permutation entropy patterns of electroencephalogram recorded during epileptiform activity. Электроника и связь, v. 19, n. 1, р. 6-14, 2014.

CARDOSO, R. P. Uma estratégia de modelagem tridimensional para mapeamento do EEG de superfície. 2005. 187 f. Dissertação (Mestrado em Ciências) - Programa de Pós-Graduação da Universidade Federal de Uberlândia, Uberlância, 2005.

CHEN, D. F; JIAO, J. Induction of neurogenesis in nonconvetional neurogenic regions of the aduly central nervous system by niche astrocyte-produced signals. Stem Cells, v. 26, p. 1221 - 1230, 2008. DAVIS H; DAVIS, P. A. The electrical activity of the brain its relation to physiological states and to states of impaired consciousness. Research Publications, Association for Research in Nervous Mental Diseases, v. 19, p. 50, 1939.

GARCIA, E. A. C. Biofísica. São Paulo: Editora Sarvier, 505 f. 2015. GREENE-SCHOLOESSER D; ROBBINS M. E; PEIFFER, A. M; SHAW, E. G. SHAW, WHEELES, K. T; CHAN, M. D. Radiatoon-induced brain injury: a review. Frontiers in Oncology, v. 2, n. 73, p. 1 - 18; 2012. IBÁÑEZ-MOLINA, A. J. ET AL. Multiscale Lempel-Ziv complexity for EEG measures. Clinical Neurophysiology, v. 126, n. 3, p. 541-548, 2015.

KASPAR F. AND SCHUSTER H.G., Easily calculable measure for the complexity of spatiotemporal patterns. Physics Review A, v. 36, no. 2, pp. 842-848, 1987.

KEDADOUCHE M., THOMAS M., TAHAN A., GUILBAULT R, Monitoring gears by vibration measurements: Lempel-Ziv complexity and Approximate Entropy as diagnostic tools, MATEC Web of Conferences 20, 2015.

LEE, CHUN SIONG ET AL. Integrating EEG Modality in Serious Games for Rehabilitation of Mental Patients. In: Simulations, Serious Games and Their Applications. Abstract... Springer Singapore. 2014. p. 51-68.

LEMPEL A. AND ZIV J., On the complexity of finite sequences. IEEE Trans. Inform. Theory, Jan. IT22, 75-81, 1976.

LOGANOVSKY, K. N; YURYEV, K. L. EEG patterns in persons exposed to ionizing radiation as a resulto the Chernobyl accident: part 1: conventional EEG analysis. The Journal of neuropsychiartry and clinical neurosciences, v. 13, n. 4, p. 441 - 458; 2001.

LOGANOVSKY, K. N. Do low doses of ionizing radiation affect the human brain? Advance Publication, Data Science Journal; jun. 2009.

MONJE, M. L; PALMER, T. Radiation injury and neurogenesis. Current opinion in neurology, v. 16, n. 2, p. 129 - 134, abr. 2003.

NUNES, C. A. J. Algoritmo de Lempel-Ziv aplicado à classificação quantitative de autômatos celulares. 2014. 127 f. Dissertação (Mestrado em Física) - Programa de Pós-Graduação em Física da Universidade Federal de Uberlândia, Uberlândia, 2014.

PESSOA, D; CRUZ, R; MACHADO, B; TENORIO B; NOGUEIRA R. A. Analysis of electrocorticographic patterns in rats fed standard or hyperpidic diets in a normal state or during status epilepticus. Nutritional Neuroscience, v. 0, n. 0, p 1 -7; 2015.

SANTOS, W. Análise estatística do eletrocorticograma durante o fenômeno da depressão alastrante em córtex cerebral de ratos nutridos e desnutridos. 2007. 88 f. Dissertação (Mestrado em 
Biometria e Estatística Aplicada). Universidade Federal de Pernambuco, Pernambuco, 2007.

SCHINEIDER, F. Uso da calendula officinalis na prevenção $e$ tratamento de radiodermite em cabeça e pescoço: ensaio clínico randomizado duplo cego. 2012. 112 f. Dissertação (Mestrado em Enfermagem) - Programa de Pós-Graduação em Enfermagem da Universidade Federal do Paraná, Curitiba, 2012.

SIMONS, S.; ABASOLO, D.; HUGHES, M.. Investigation of Alzheimer's Disease EEG Frequency Components with Lempel-Ziv Complexity. In: 6th European Conference of the International Federation for Medical and Biological Engineering. 2015. Abstract... Springer International Publishing, 2015. p. 46-49.

WEISSTEIN, E. W. Fourier Transform. MathWorld, A Wolfram Web Resource, 2004.

YAAR I; RON E; MODAN B. Long-lasting cerebral functional changes following moderate dose $\mathrm{X}$-radiation treatment to the scalp in childhood: an EEG power spectral study. Journal of Neurology Neurosurgery and Psychiatry, v. 45, p. 166 - 169; 1982. 